\title{
VALORACIÓN DE LOS DERECHOS HUMANOS EN UN GRUPO DE ESTUDIANTES UNIVERSITARIOS DE LIMA METROPOLITANA
}

\author{
Ramón León ${ }^{1}$ / Eduardo Gamarra Alarco²
}

\section{Resumen}

518 universitarios (262 hombres y 256 mujeres, entre los 16 y 33 años de edad) residentes en Lima Metropolitana respondieron una encuesta de 27 items que tenía por objetivo evaluar su valoración de los derechos humanos. El análisis factorial arrojó cuatro factores: Autoritarismo, Punición severa, Vigilancia y represión; y Restricción de derechos. La muestra se mostró favorable a la implantación de la pena de muerte y rechazo a la legalización del aborto.

Palabras Clave: Derechos humanos - Universitarios - Lima Metropolitana .

\section{Abstract}

518 students (262 men and 256 women, 16-33 years old) living in Metropolitan Lima answered a 27-items survey about their valuation of human rights. Factorial analyzed results showed four factors: Authoritarianism, Severe Punition, Vigilance and Repression; and Restricted Rights. The students were for the introduction of death penalty and against the legalization of abortion.

Key words: Human rights - Students - Metropolitan Lima. 
Los derechos humanos tienen mala prensa en el Perú. Desde los años de la lucha contra el terrorismo hasta la actualidad, muchas acciones y decisiones tomadas por el estado peruano en respeto a los derechos humanos en el trato, enjuiciamiento y condena de terroristas, han sido criticadas por buena parte de la prensa televisiva, radial y escrita, pero también por quienes consideran que el estado no asume una política decidida y de mano fuerte no solo en lo que se refiere a los terroristas sometidos a juicio, sino también a delincuentes comunes cuyas fechorías han impactado a la opinión pública.

Así, por ejemplo, el informe de la Comisión de la Verdady Reconciliación, dado a conocer en 2003, recuento e interpretación de lo sucedido durante los años del terrorismo en el Perú, en lugar de haber sido recibido con interés, generó más bien numerosas críticas que aún continúan e inclusive han alcanzado en los últimos tiempos un tono particularmente agresivo (Walker 2009). Con motivo de cumplirse los diez años de la aparición del informe las criticas han continuado, si bien hay posiciones de apoyo y defensa del mismo (véase, sobre el particular, entre otros, Lay Sun 2013, Lerner Febres 2013, Meléndez 2013, Santillana 2013, Santiváñez Vivanco 2013; también Pease García \& Romero Sommer 2013).

Pero ha habido otra actitud, tal vez más grave: la de la indiferencia por parte de la gran mayoría de la sociedad peruana que manifestó un desconcertante desinterés frente al trabajo de la Comisión de la Verdad, unida a un desconocimiento casi absoluto del contenido del informe y de las conclusiones del mismo.

Esto está en crasa contradicción con las reacciones de muchas personas ante temas que pueden considerarse nimios o hasta banales. Las afirmaciones, formuladas no hace mucho, por un escritor peruano acerca de la gastronomía local, calificándola de indigesta y manifestando su poca preferencia por ella, despertaron reacciones airadas en la radio, en la televisión, en los twitter, descalificándolo muchas veces con frases ofensivas, y defendiendo la calidad y el gusto de la cocina peruana con una energía digna de mejor causa (Noticia 2012).

Algo semejante puede decirse con respecto a lo sucedido con la Corte Interamericana de los Derechos Humanos, en la cual el Perú tiene numerosas demandas pendientes, y cuyas resoluciones no siempre han sido del agrado del gobierno y de la prensa peruanas, habiéndose reclamado en varias ocasiones $\mathrm{y}$ diversos contextos el retiro del Perú de dicha corte.

Por si fuera poco, muchas figuras destacadas de la politica peruana se han manifestado de manera despectiva acerca de los derechos humanos, su vigencia y su aplicación. Al lado de las declaraciones del presidente Alan García solicitando, en su segundo gobierno, la reintroducción de la pena de muerte para violadores (Noticia 
2010), es posible encontrar a una serie de personalidades para las cuales los derechos humanos son casi algo innecesario.

El presente trabajo tiene por finalidad explorar las actitudes frente a los derechos humanos en general en un grupo crítico de la sociedad peruana, los estudiantes universitarios.

\section{MARCO TEÓRICO}

Aunque Ishay (2004) encuentra antecedentes referidos a ellos ya en la obra de Bartolomé de las Casas y en la de Hugo Grocio, lo cierto es que los derechos humanos tienen una existencia nominal desde la Revolución Francesa, que los proclamó en 1790 , y reflejan mucho de la comprensión que hemos desarrollado acerca de la condición humana (Pirttilä et al. 2009). Pero desde entonces hasta su plena aceptación y su decidida defensa en nuestros días han pasado muchos años. Moghaddam \& Vuksanovic (1990) señalaban que los derechos humanos representan uno de los más importantes desafios que afrontan las sociedades contemporáneas.

El concepto contemporáneo de derechos humanos, relativamente nuevo en la historia de la humanidad (DíazVeizades et al., 1995), sugiere ciertas libertades o prerrogativas fundamentales que todos los individuos deberían tener en la misma proporción (Moghaddam \& Vuksanovic 1990).

Se habla en la actualidad de derechos humanos de diferentes generaciones. Los de primera generación surgen en el siglo XVIII y XIX y son sobre todo derechos cívicopolíticos; los de segunda generación (derechos sociales, económicos, culturales) se forjan en el siglo XIX; la tercera generación de ellos (derecho al desarrollo, autodeterminación) surgen en el siglo XX, así como la cuarta generación (derechos de los pueblos indígenas) (Messer 2002).

Kinderman (2007) resume los principales derechos tal como han sido presentados en la Human Rights Act de 1998 en el Reino Unido: derecho a la vida, prohibición de torturas y tratamientos inhumanos o degradantes, prohibición de esclavitud y de trabajos forzados, derecho a la libertad y seguridad de la persona, derecho a un debido proceso, prohibición de criminalización retroactiva, derecho a la vida familiar y privada, el hogar y la correspondencia, libertad de pensamiento, conciencia y religión, libertad de expresión, libertad de reunión y de asociación, derecho a casarse y formar una familia, derecho a no ser objeto de discriminación en el disfrute de los otros derechos.

La actual preocupación por los derechos humanos fue estimulada por la conciencia que la humanidad tomó de las terribles circunstancias, maltratos y genocidio de las que fueron víctimas quienes vivieron bajo la férula del nacionalsocialismo y del stalinismo, pero también los que sufrieron dictaduras como las de España (bajo Franco, 19381975), Paraguay (bajo Stroessner, 
1954-1989), Argentina (bajo Videla y compañia, 1976-1983) y, más recientemente, Chile (bajo Pinochet, 1973-1990).

Bolivar \& Cubas (2009) ubican a fines de la década de los 1960 el surgimiento de las organizaciones no gubernamentales de derechos humanos tales como Amnesty International y Human Rights Watch.

Un rasgo central de los derechos humanos es la irreversibilidad de los mismos: es decir, no pueden ser revertidos ni suprimidos, dado que constituyen logros de la humanidad en su camino a un mundo digno.

El interés por los derechos humanos a nivel internacional queda expresado en el hecho de que el número de organizaciones dedicadas a la educación de los mismos se ha cuadruplicado entre 1980 y 1995 y sigue aumentando (Ramírez et al. 2006, Suarez \& Ramírez 2007).

Otro indicador de la preocupación internacional se encuentra en un estudio que examinó los reportes de diferentes países al International Bureau of Education (IBE) y al Alto Comisionado de las Naciones Unidas para los Derechos Humanos: el número de veces que el término derechos humanos fue mencionado en los documentos oficiales remitidos por los países a la IBE fue calculado considerándolo como un indicador del interés de cada estado por el tema (Ramirez et al. 2006). El resultado en este indicador fue de .82 para Europa del Este y la antigua Unión Soviética, .70 para países en el Africa subsahariana, .64 para los países en América Latina y el Caribe, y .11 para los países de Europa Occidental y de América del Norte.

Finalmente, la educación en derechos humanos es crecientemente reconocida como un rasgo distintivo importante de la educación ciudadana (Howe \& Cowell 2005) y una de las bases de sociedades democráticas y pluralistas (Bravc et al. 2011).

En el Perú, el tema de los derechos humanos cobra fuerza a partir de los años del terrorismo. La cruenta actividad de Sendero Luminoso (de 1980 a 1992), con su impresionante secuela de muertos, desaparecidos y mutilados, fue respondida por el estado con una ofensiva militar que no siempre distinguió entre la población civil y los terroristas (Burt 2006, 2010). La magnitud de la violencia política en el país superó largamente a la de Ecuador y Bolivia (Imsbuch et al. 2011).

Los sangrientos acontecimientos de aquel entonces trajeron por tierra la difundida idea de que "el peruano era una persona pacífica”, y, más bien, nos confrontaron con una sociedad violenta y autoritaria, cuya expresión más espantosa era Sendero Luminoso (Manrique 1996, Portocarrero 1990, 1993)

En los años del terrorismo y de la lucha del estado en contra de él se perpetraron hechos que constituyen violaciones de los derechos humanos, que después han sido registrados $\mathrm{y}$ denunciados por diferentes ONGs y por el Informe de la Comisión de la 
Verdad y la Reconciliación.

Desde entonces, como decimos, aparece entre nosotros la insistencia en la defensa y el respeto a los derechos humanos, que, sin embargo, no siempre han sido bien recibidos por la sociedad peruana, pues en ella, como en otras sociedades que han sufrido procesos de violencia política, se suele ver dicha defensa por parte de las ONGs como políticas de protección para terroristas, pues insisten en el debido proceso y en la presunción de inocencia hasta la demostración de la culpabilidad (Cesarini \& Hertel 2005).

Pero no solo el terrorismo ha sido uno de los factores detonantes para una actitud de la opinión pública por lo general negativa frente a la implementación y respeto de los derechos humanos. También lo ha sido el rasante incremento de la delincuencia y de los crimenes: de hecho América Latina ocupa el segundo lugar en el ranking de homicidios a nivel mundial (Humphrey 2012).

Los elevados niveles de violencia cotidiana (robos, asaltos, asesinatos, etc.), las penas consideradas en muchos casos como benévolas para muchos de los terroristas y delincuentes capturados y debidamente juzgados, la imposibilidad de condenar a la pena de muerte a quienes cometen asesinatos que generan la indignación de la población peruana (por ejemplo, casos de incesto y asesinato), han dado lugar a una demanda de "una mano dura" y de medidas draconianas que incluyan la pena de muerte (Iturralde 2010).

$\mathrm{Si}$ bien la demanda por la reintroducción de la pena de muerte está muy difundida pero no necesariamente tiene una consistencia firme entre la población (Sarat \& Vidmar 1976), el hecho de que se la formule supone un desafío dramático a la corriente de los derechos humanos.

El Informe Anual 2010 sobre Seguridad Ciudadana preparado por Lovatón Palacios y Arias Aróstegui (2010) para el Instituto de Defensa Legal, señaló que el $26 \%$ de los encuestados era de la opinión que la causa de la inseguridad ciudadana en Lima y El Callao radicaba en la "poca dureza de las penas". Cuando los encuestados fueron preguntados acerca de las tres principales medidas que debían tomarse para combatir la delincuencia ese porcentaje se elevó a $34 \%$.

Esto no es algo exclusivo de la sociedad peruana. En los Estados Unidos, como consecuencia del ataque terrorista perpetrado en el World Trade Center, se observa en la población una corriente de aprobación a medidas de fuerza que ha emprendido el gobierno norteamericano, algunas de las cuales afectan los derechos humanos (Brooks \& Manza 2013). Las dictaduras en Chile y Argentina justificaron el terrorismo de estado que llevaron a cabo como una medida extrema de lucha contra lo que llamaron enemigos de la democracia $\mathrm{y}$, asimismo, encontraron aprobación en parte de la opinión pública (Wright 2007).

Como lo señala el International Council on Human Rights Policy 
(2003), "cuando la delincuencia se convierte en un problema, se observa con frecuencia la emergencia de una constante: ante una delincuencia en alza aumenta asimismo el apoyo público a las políticas de orden público duras" (pg. 2). Algo semejante anotan Rico \& Chinchilla (2006).

Eso es lo que ha venido ocurriendo en el Perú, en el cual la percepción de inseguridad ha ido en constante aumento. Ya hace más de diez años, Reyna \& Toche (1999) afirmaban que "en términos generales [...] tenemos que la percepción ciudadana sobre la inseguridad de su entorno ha ido aumentando en la medida que estima que aumentan los delitos, pero también porque se mantienen o aumentan la ineficacia o la corrupción de las instituciones que deben garantizar el orden público" (pg. 13).

Véase, para datos recientes, la VIII Encuesta Nacional sobre percepciones de la corrupción en el Perú 2013, basada en 1202 entrevistados, que encuentra que la delincuencia es uno de los problemas más mencionados (Total: 63\%; NSE A: 63\%; NSE B: $66 \%$; NSE C: $62 \%$; NSE D: $62 \%$; y NSE E: 62\%). Un estudio igualmente reciente del costo económico de la delincuencia organizada en el Perú señala que "en los últimos años la delincuencia, incluyendo el robo agravado, el homicidio, el secuestro, la violación sexual, y el pandillaje, ha pasado de $10 \mathrm{mil}$ casos en el 2007 a 12 mil en 2009; es decir, muestra un ritmo de crecimiento promedio de $11 \%$ anual", agregando que "los registros de detenciones policiales casi se duplicaron en esos dos años $(92 \%)$, junto al número de bandas delincuenciales $(96 \%)$ que superó las 2 mil" (Mirella 2011, pg. 8).

$\mathrm{Si}$ bien un reciente informe del estado peruano señala que el nivel de victimización ha experimentado un descenso en los últimos tiempos (Ministerio de Justicia y Derechos Humanos 2013), la percepción de la inseguridad ciudadana continúa siendo muy elevada.

Cuando el presidente Alan García propuso la reimplantación de la pena de muerte, encontró una favorable acogida entre los peruanos, como lo señala Apoyo. Opinión y Mercado (2007): "la pena de muerte siempre ha despertado entusiasmo en sociedades con elevada inseguridad ciudadana y un bajo nivel educativo. El Perú no podía ser la excepción. En realidad, mucha gente quisiera que se ejecute no solo a violadores y terroristas sino también a los responsables de los más diversos delitos. En ese contexto, García ha logrado persuadir a la opinión pública de que su empecinamiento a favor de la pena de muerte es consecuencia de una preocupación sincera por mejorar la seguridad de la población” (pg. 1).

En otros casos, que han impactado a la opinión pública, muchas personas se han tomado la justicia por sus manos, y esto en lugar de generar rechazo ha despertado una ola de aceptación.

En el trasfondo de lo anteriormente descrito podemos reconocer, además, 
a una sociedad caracterizada por un arraigado autoritarismo y, consecuentemente, por una valoración muy baja de la democracia y de las formas que ésta tiene de resolver conflictos sociales y de sancionar a quienes cometen infracciones y delitos.

El autoritarismo de la sociedad peruana es, sin duda, una de sus características más acusadas, y ha sido estudiado por diferentes autores (por ejemplo, Flores Galindo 1999; y Torres Arancivia 2003).

A pesar del paso del tiempo, este rasgo no ha disminuido en su intensidad. Por el contrario, podría decirse que se ha acentuado. El gobierno de Alberto Fujimori fue uno de los más autoritarios de los últimos decenios y a él se le imputa un elevado número de casos de violación grave de los derechos humanos (Burt 2006, 2010). El presidente García en su segundo gobierno dio también repetidas muestras de intolerancia contra la crítica (aun en casos en los cuales ésta era expresada de manera alturada), recurriendo a la descalificación personal y al insulto abierto (León 2010) y estimulando al mismo tiempo una corriente de caudillismo claramente reconocible.

Burt (2006), tratando de explicar la tendencia al autoritarismo y a la aplicación de la mano dura, señala que la violencia política vivida en el Perú "ha alimentado la desilusión popular con respecto a las normas y procedimientos democráticos, haciendo que las soluciones autoritarias aparezcan más aceptables" (pg. 42).

\section{PROBLEMA}

Mucho se ha hablado sobre esto, pero existe poca información fáctica. Poco se sabe además de lo que piensan los adolescentes y jóvenes acerca de la importancia de los derechos humanos, la necesidad de su promoción y respeto en la sociedad peruana, y acerca de algunos de los temas que están incluidos en ellos (como, por ejemplo, el respeto a la orientación sexual de las personas, el respeto a la prensa, el derecho al aborto).

El desconocimiento sobre el particular en el Perú contrasta con la información que es posible encontrar en la literatura internacional.

Así, por ejemplo, Torney-Purta \& Wilkenfeld (2008) llevaron a cabo un análisis de la información proveniente de 88 mil estudiantes en edad de 14 años encuestados en el International Association for the Evaluation of Educational Achievement (IEA) Civic Education Study en 1999. América Latina estuvo representada en este estudio por Chile y Colombia. Los resultados permiten afirmar que países con gobiernos que conceden más atención a los derechos humanos en los discursos intergubernamentales (tales como por ejemplo el diálogo entre naciones $\mathrm{y}$ organismos gubernamentales internacionales) tenían estudiantes que obtenían más elevados puntajes en los ítems de conocimientos de dichos derechos. Asimismo, los estudiantes que tenian en sus colegios experiencias democráticas y con temas internacionales tenían una vinculación positiva 
con el conocimiento de los derechos humanos.

Moghaddam \& Vuksanovic (1990) encontraron en un estudio efectuado en Canadá que personas que eran más religiosas y con una orientación de derecha tendian a ser más opuestas a los derechos humanos en los contextos canadienses y del Tercer Mundo, pero esa tendencia no se mantenía cuando se trataba del contexto soviético.

En el presente trabajo nos proponemos explorar estos y otros aspectos en una muestra de adolescentes y jóvenes universitarios.

Conocer sobre el particular es algo necesario dado que los adolescentes y jóvenes de hoy son los ciudadanos que en pocos años influirán, con sus opiniones, actitudes y votos, en las decisiones politicas del país y, a su vez, educarán a la siguiente generación en valores y formas de pensar sobre temas de relevancia en el plano político, ético y social.

La reducción de la pobreza en el Perú y el aumento de la así llamada clase media convierte a ésta en un tema de interés para la investigación sociológica y psicológica. Representante de valores tales como el emprendimiento, el ahorro sistemático y la búsqueda de ascenso social, la clase media sin embargo suele ser presentada también como muy preocupada por el orden social y por la necesidad de contar con políticas que garanticen la posibilidad de su desarrollo, aun cuando esto vaya asociado a que se adhiera o muestre poco rechazo ante prácticas autoritarias (Ozbudun 2005,
Tiano, 1986, Paramio 2011, Lipset 1959).

Por ese motivo, nuestro estudio buscó asimismo conocer las actitudes de adolescentes y jóvenes de clase media sobre el particular.

\section{MATERIAL Y MÉTODO}

Se elaboró una escala tipo Likert con 27 items (con 7 posibilidades de respuesta, desde totalmente de acuerdo hasta totalmente en desacuerdo), que evalúaban las actitudes frente a algunos de los derechos humanos en su aplicación a quienes cometen delitos (desde delitos en general hasta violaciones), a la necesidad de actuar con mano dura por parte del estado, a las politicas de beneficios para los delincuentes, con respecto al terrorismo y a la actuación de ONGs dedicadas a defender los derechos humanos (véase el apéndice).

Asimismo, se pidió a los sujetos de la muestra que se ubicaran en una escala de clase social (clase alta, clase media y clase baja) y que se autocalificaran en términos de su orientación politica, de izquierda a derecha.

Esta escala fue aplicada a una muestra de estudiantes de una universidad privada de Lima Metropolitana (cuyos costos de enseñanza la ubican como posible de ser elegida por estudiantes de los niveles socioeconómicos A, B y C) de manera anónima y colectiva contando con la autorización de ellos para ser evaluados. La muestra estuvo conformada por 518 estudiantes (262 hombres y 256 mujeres), entre los 16 y 33 años de edad. 


\section{RESULTADOS}

Con referencia a la clase social, $1.5 \%$ (8 personas) se consideró de clase alta, $93.8 \%$ (486) de clase media, y $4.6 \%$ (24) de clase baja.

En materia de orientación política, en la que se consideraron 9 posibilidades, la tabla 1 da a conocer los resultados.

Tabla 1: Orientación política autovalorada de los estudiantes ( $N=518)$

\begin{tabular}{|c|c|c|}
\hline Orientación politica & $\mathbf{N}$ & $\%$ \\
\hline 1 Extrema Izquierda & 18 & 3.5 \\
\hline $\mathbf{2}$ & 4 & .8 \\
\hline $\mathbf{3}$ & 21 & 4.1 \\
\hline $\mathbf{4}$ & 34 & 6.6 \\
\hline $\mathbf{5}$ Centro & 215 & 41.5 \\
\hline $\mathbf{6}$ & 57 & 11.0 \\
\hline $\mathbf{7}$ & 43 & 8.3 \\
\hline $\mathbf{8}$ & 20 & 3.9 \\
\hline 9 Extrema Derecha & 59 & 11.4 \\
\hline No respondió & 47 & 9.1 \\
\hline Total & 518 & 100 \\
\hline
\end{tabular}

Como puede observarse, la mayoría de los sujetos se ubicó en el centro $(41.5 \%)$, en la extrema izquierda se ubicaron solo 18 (3.5\%) y en la extrema derecha 59 (11.4\%).

La escala de actitudes hacia los derechos humanos, por su parte, mostró características estadísticas apropiadas. El nivel de correlación ítem-total de todos los items fue de .26 para arriba, excepto el 21 ("A veces es necesario el castigo físico en la escuela"). Dos items tuvieron una correlación item total negativa (14, "La libertad de prensa debe ser total": -.314); y, 16, ("Se debe legalizar el aborto en el Perú": -.216). El alfa de
Cronbach para toda la escala fue de .83 (véase la tabla 2).

Tabla 2: Promedios y desviación standard de cada item

\begin{tabular}{|c|c|c|}
\hline Item & M & DS \\
\hline 01. & 4,72 & 1,384 \\
\hline 02. & 5,60 & 1,658 \\
\hline 03. & 4,43 & 1,449 \\
\hline 04. & 3,64 & 1,444 \\
\hline 05. & 5,50 & 1,341 \\
\hline 06. & 4,87 & 1,658 \\
\hline 07. & 5,98 & 1,118 \\
\hline 08. & 5,33 & 1,210 \\
\hline 09. & 5.57 & 1,332 \\
\hline 10. & 4,90 & 1,306 \\
\hline 11. & 3,76 & 1,882 \\
\hline 12. & 4,65 & 1,272 \\
\hline 13. & 5,14 & 1,413 \\
\hline 14. & 2,97 & 1,489 \\
\hline 15. & 3,71 & 1,847 \\
\hline 16. & 4,25 & 1,912 \\
\hline 17. & 5,32 & 1,728 \\
\hline 18. & 5,37 & 1,449 \\
\hline 19. & 5,38 & 1,249 \\
\hline 20. & 3,07 & 1,703 \\
\hline 21. & 2,53 & 1,658 \\
\hline 22. & 5,86 & 1,247 \\
\hline 23. & 5,86 & 1,171 \\
\hline 24 & 5,00 & 1,377 \\
\hline 25. & 5,24 & 1,837 \\
\hline 26. & 5,83 & 1,271 \\
\hline 27. & 6,04 & 1,278 \\
\hline
\end{tabular}

Los más altos promedios los obtuvieron los ítems 27 ("los delincuentes tienen demasiados derechos en el Perú-por ejemplo, reducción de pena", 6.04), 7 ("en el Perú se debe tener mano dura para controlar el desorden social"; 5.98) y el 22 ("el orden es lo más importante en una sociedad", 5.86) y el 23 ("la autoridad siempre debe ser respetada", 5.86) . 
Los más bajos correspondieron al item 21 ("A veces es necesario el castigo fisico en la escuela"; 2.53), 14 ("La libertad de prensa debe ser total en el Perü"; 2.97); y, 20 ("El castigo físico de los hijos por parte de sus padres es un derecho de éstos, y el estado no debe intervenir"; 3.07).
A continuación se procedió a llevar a cabo un análisis factorial exploratorio (AFE), mediante el análisis de componentes principales y empleando como método de rotación la normalización Varimax con Kaiser. El gráfico 1 presenta el grado de sedimentación

Gráfico 1: Sedimentación del AFE

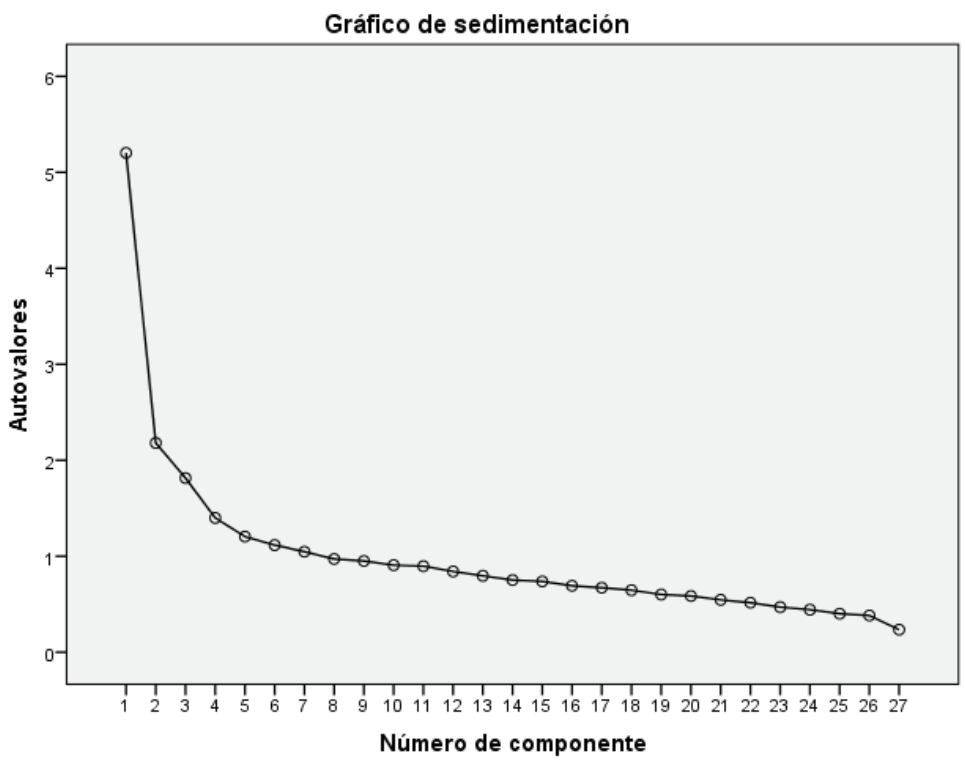

El análisis factorial arrojó 7 componentes, que explicaron el 51\% de la varianza, pero se generaban factores con pocos ítems. Por ello, se decidió, a fin de obtener una precisión y concentración de los factores, un nuevo AF con 4 factores identificados a partir del análisis del gráfico de sedimentación. Los resultados de la rotación final son presentados a continuación. 
Tabla 3: Resultados del análisis factorial con 4 factores

\begin{tabular}{|c|c|c|c|c|}
\hline Ítem & 1 & 2 & 3 & 4 \\
\hline 01 & & & & ,299 \\
\hline 02 &, 565 & &,-390 & \\
\hline 03 & & & &,- 352 \\
\hline 04 & & ,327 & & \\
\hline 05 & ,316 & & ,453 & \\
\hline 06 & ,437 & & & \\
\hline 07 & ,655 & & & \\
\hline 08 & ,463 & & ,318 & \\
\hline 09 &, 583 & & & \\
\hline 10 & ,435 & & & \\
\hline 11 & & &, 570 & \\
\hline 12 & ,409 & & ,417 & \\
\hline 13 & ,447 & & & \\
\hline 14 & & & & ,422 \\
\hline 15 & & & &, 583 \\
\hline 16 & &,- 383 & & ,330 \\
\hline 17 &, 575 & &,-319 & \\
\hline 18 & ,497 & & & \\
\hline 19 &, 590 & & & \\
\hline 20 & & ,691 & & \\
\hline 21 & & ,701 & & \\
\hline 22 & ,491 & & & \\
\hline 23 &, 503 & & & \\
\hline 24 &, 534 & & & \\
\hline 25 &, 576 & ,323 &,-414 & ,320 \\
\hline 26 & ,677 & & & \\
\hline 27 & ,612 & & & \\
\hline Eigenvalue & 5.202 & 2.180 & 1.815 & 1.399 \\
\hline$\%$ var.explicado & 19.267 & 8.074 & 6.721 & 5.181 \\
\hline
\end{tabular}

Método de extracción: Análisis de componentes principales. ${ }^{a}$

Los cuatro factores hallados explicaron el $39.24 \%$ de la varianza. A fin de determinar la fiabilidad de cada uno se calculó el alpha de Cronbach encontrándose coeficientes aceptables: .77 para el factor 1 (Autoritarismo; 18 items); .75 para el factor 2 (Punición severa; 5 items); .71 para el factor 3 (Vigilancia y represión; 7 items); y,
.68 para el factor 4 (Restricción de derechos; 6 items).

La tabla 4 presenta la correlación de Spearman de los cuatro factores. Como puede verse, la correlación entre el factor 1 y 2 es moderada (,569; $<.01$ ), en tanto que las de los demás son bajas. 
Tabla 4: Correlaciones de los cuatro factores

\begin{tabular}{|c|c|c|c|c|}
\hline Factor & Factor 1 & Factor 2 & Factor 3 & Factor 4 \\
\hline 2 &, $569^{* * *}$ & ---- & & \\
\hline 3 &, 041 &, 022 & --- & \\
\hline 4 &, 156 &, 024 &, 069 & ---- \\
\hline
\end{tabular}

\section{Clase social}

En el factor 1 el promedio para la clase alta fue 52.87, en tanto que el de la clase media fue 55.00 y el de clase baja 52.91. En el factor 2 la clase alta obtuvo el promedio más elevado (44.88) seguida por la clase media (42.57) y la clase baja (40.63). En el factor 3 fue más bien la clase baja la que obtuvo el promedio más alto (14.88) seguida por la clase media 12.69) y la clase alta (12.13). Por último, en el factor 4 el promedio más elevado, 22.28, correspondió a la clase media, en tanto que la clase baja tuvo un promedio de 20.96 y la clase alta 20.75.

Dado que los resultados no se ajustaron a la curva de distribución normal se procedió a aplicar la prueba de la mediana, encontrándose diferencias significativas en el factor 1 $(\mathrm{X} 2=9.616 ; 008), 2(\mathrm{X} 2=6.945 ; .031) \mathrm{y}$ $4(\mathrm{X} 2=6.582 ; .037)$ (ver tabla 5$)$.

Tabla 5: Prueba de la mediana de clase social

\begin{tabular}{|l|r|r|r|r|}
\hline & \multicolumn{1}{|c|}{ FACTOR1 } & FACTOR2 & \multicolumn{1}{|c|}{ FACTOR3 } & \multicolumn{1}{|c|}{ FACTOR4 } \\
\hline Mediana & 55,000 & 44,00 & 13,00 & 22,00 \\
\hline Chi-cuadrado & 9,616 & 6,945 & 3,879 & 6,582 \\
\hline g1 & 2 & 2 & 2 & 2 \\
\hline Sig. asintót. &, 008 &, 031 &, 144 &, 037 \\
\hline
\end{tabular}

\section{Orientación política}

En lo concerniente a la orientación política, en el factor 1 el promedio más alto correspondió a los que se clasificaron como de derecha (56.08), mientras que los de izquierda tuvieron como promedio 55.44 y los de centro 54.30. En el factor 2, los de derecha una vez más obtuvieron el promedio más elevado (44.25), seguidos por los de la izquierda (43.65) y los del centro (41.66). En el factor 3 fue, más bien, el grupo que se autodefinió como de izquierda el que obtuvo el promedio más elevado (14.23), seguida por los de centro y los de derecha (12.78 y 12.34, respectivamente). Finalmente, en el factor 4 el promedio más alto correspondió a la derecha (22.39) en tanto que la izquierda y el centro obtuvieron promedios casi iguales (22.12 y 22.11, respectivamente).

Como los resultados, asimismo, no se ajustaron a la curva de distribución normal se procedió a aplicar la prueba de la mediana, encontrándose diferencias significativas en los factores $2(\mathrm{X} 2=11.477 ; .003)$ y $3(\mathrm{X} 2=$ 7.488.024). 
Tabla 6: Prueba de la mediana de orientación politica

\begin{tabular}{|l|r|r|r|r|}
\hline & \multicolumn{1}{|c|}{ FACTOR1 } & FACTOR2 & \multicolumn{1}{|c|}{ FACTOR3 } & \multicolumn{1}{c|}{ FACTOR4 } \\
\hline Mediana & 55,000 & 44,00 & 13,00 & 22,00 \\
\hline Chi-cuadrado & 4,932 & 11,477 & 7,448 & 1,518 \\
\hline gl & 2 & 2 & 2 & 2 \\
\hline Sig. asintót. &, 085 &, 003 &, 024 &, 468 \\
\hline
\end{tabular}

\section{DISCUSIÓN}

\section{Items con los promedios más elevados}

Los tres items que obtuvieron los promedios más elevados insistieron en el hecho de que los delincuentes tienen demasiados derechos (item 27) y que el orden debe ser preservado al mismo tiempo que la autoridad debe ser respetada (7 y 22).

No es exagerado suponer que la mayor puntuación en estos ítems está vinculadacon la creciente conflictividad social en el Perú de los últimos años, en la cual la toma de carreteras, la omisión del cumplimiento de normas dictadas por el gobierno (como el estado de emergencia en Cajamarca con referencia al conflicto de Conga), la resistencia activa a cumplir mandatos judiciales y la creciente criminalidad, han dado lugar a que los medios de comunicación oral, escrita y televisiva insistan a través de comentarios y editoriales en la necesidad de preservar el orden y restablecer el principio de autoridad.

La inseguridad ciudadana in crescendo, a pesar de la afirmación de las autoridades en un sentido contrario, así como los escándalos derivados del hecho de que en muchas ocasiones los delincuentes capturados han sido liberados por dictámenes judiciales, explican la insistencia de los sujetos de la muestra en que los delincuentes tienen demasiados derechos y que es necesario reformar algunas normas jurídicas a fin de endurecer las penas y reducir los beneficios penitenciarios de los que disponen.

\section{Items con los promedios más bajos}

Por su parte, los tres ítems con los promedios más bajos estuvieron referidos al castigo físico en la escuela y en el hogar (items 20 y 21) y en la aceptación de que la prensa goce de libertad total en el Perú (14).

El rechazo del castigo físico como instrumento de socialización es una de las tendencias más difundidas en el mundo moderno y corresponde a una mentalidad más abierta, democrática y tolerante en las prácticas de crianza de la clase media (Panez 1989, Majluf 1989, Majluf \& Ojeda 2006).

En lo que se refiere a una irrestricta libertad de prensa puede suponerse que la escasa aprobación de ella tiene que ver con los frecuentes reportes periodísticos acerca de situaciones anómicas, con escándalos o situaciones escabrosas en las que el periodismo hurga de manera insistente e incisiva, y con el desprestigio que muchos medios de comunicación experimentaron al momento en que 
cayó el régimen de Fujimori y se descubrieron casos en los cuales se había pagado a periodistas y a órganos de prensa a fin de que informaran (o desinformaran) a favor del gobierno.

\section{Análisis factorial}

El primer factor, Autoritarismo, refleja una actitud favorable hacia un estado fuerte, que aplique mano dura y haga prevalecer el orden, utilizando medidas radicales (como la pena de muerte). Este factor refleja además una actitud de escepticismo con respecto a la democracia y a sus instituciones (el parlamento, por ejemplo, pero es de suponer que también el poder judicial, dos de los poderes con el más elevado nivel de desprestigio; vide Noticia 2013, en la que se señala que en abril del 2013 el 75\% y el 73\%, respectivamente, de 1321 encuestados los desaprobaban).

Este factor expresa la mentalidad autoritaria prevalente en los integrantes de la muestra, intensificada por el desorden social provocado por los conflictos socio-ambientales, pero también por la delincuencia cada vez mayor.

Dicha mentalidad está profundamente arraigada en la sociedad peruana, cuya experiencia con prácticas democráticas es muy limitada (Flores Galindo 1999, Torres Arancivia 2003, Walker 2009, Pease \& Romero Sommer 2013).

El segundo factor, Punición severa, refleja actitudes favorables hacia condenas drásticas (pena de muerte), pero al mismo tiempo el rechazo al castigo físico de los niños tanto en el hogar como en la escuela, como también el rechazo a la legalización del aborto.

El nivel de correlación de este factor $\mathrm{y}$ el anterior permite afirmar una estrecha relación entre ambos. El empleo de castigos severos y de medidas sumamente duras de represión es una expresión de autoritarismo.

E1 tercer factor, Vigilancia $y$ represión, comprende la creencia de que los peruanos abusan de los derechos que les concede la democracia, la necesidad del estado de controlar los periódicos, la radio y la televisión, y la aplicación de penas severas (que incluyen la pena de muerte).

Por último, el cuarto factor, Restricción de derechos, enfatiza la supuesta incapacidad de los peruanos para vivir en democracia, la creencia en una excesiva insistencia en los derechos humanos, y actitudes negativas hacia el aborto, la legalización de las uniones del mismo sexo y la libertad de prensa.

\section{Clase social}

El promedio más elevado de quienes se autoclasificaron como de clase media en autoritarismo y restricción de derechos puede ser interpretado como reflejo de una ideología muy conservadora, radicalizada por la situación de inseguridad ciudadana que se vive en el Perú de hoy, y que reclama de las autoridades mano dura y el mantenimiento del orden a cualquier precio, así como la imposición de condenas severas y aleccionadoras. De otro lado, esas personas se mostraron poco 
afectas a una prensa que posea demasiadas libertades $\mathrm{y}$, asimismo, poco tolerante también con respecto a las uniones del mismo sexo y a la legalización del aborto.

Quienes se consideraron de clase alta (con el promedio más alto en el factor 2, punición y castigo) pusieron de manifiesto asimismo tendencias sumamente conservadoras con respecto al orden social y a la necesidad de mantener un claro principio de autoridad.

Interesante es anotar, asimismo, que el factor 1, autoritarismo, en el cual los promedios de la clase media son más elevados, incluye la demanda por la pena de muerte (item 2, que está presente asimismo en los demás factores). Esa demanda se expresa en el promedio elevado que obtiene en este estudio $(5,60)$.

Aunque hay criticas a la posibilidad de evaluar las actitudes frente a la pena de muerte con solo un ítem (O'Neil et al. 2004), como lo hemos hecho, no puede dejar de anotarse este hecho. Como ya se ha dicho, las actitudes frente a la pena de muerte ofrecen una panorámica muy compleja que, como tal, demanda un análisis y estudio más detenidos. Así, por ejemplo, Baik (2012) encontró que los latinos católicos en Estados Unidos apoyaban más la pena de muerte, algo que tal vez pueda generalizarse a una población como la peruana, predominantemente católica.

\section{Orientación política}

En lo que concierne a la orientación política se encontraron diferencias significativas en los factores 2 y 3 . En el factor 2, quienes se consideraron de derecha obtuvieron el promedio más elevado revelando una mayor tendencia a la imposición de castigos severos y al uso de la mano dura, mientras que en lo que se refiere al factor 3 fueron quienes se consideraron de izquierda los que obtuvieron el promedio más elevado.

Anotemos, sin embargo, que los resultados en este punto deberían ser tomados con relativa prudencia, dado que los conceptos de izquierda y derecha si bien son muy difundidos, no siempre están claramente precisados entre muchas personas adolescentes y jóvenes, inclusive cursando estudios universitarios.

\section{COMENTARIO FINAL}

Es un hecho que en la clase baja las prácticas discriminatorias, el castigo físico, y la mentalidad autoritaria están aún muy difundidas, como lo demuestran investigaciones a lo largo del tiempo y en diferentes lugares (Lipset 1960, Nettelbladt et al. 1981, Shiraev \& Sobel 2006).

Es de destacar, sin embargo, que precisamente las clases sociales más educadas y expuestas a las tendencias modernizadoras del mundo de hoy, como son la clase alta y la media, presenten promedios elevados en autoritarismo y punición severa.

Sociedades muy desiguales, como es el caso de la peruana, tienen niveles de criminalidad más altos, prácticas y actitudes democráticas menos 
difundidas, menos solidaridad entre sus integrantes y un nivel más alto de patologías tanto fisicas como psicológicas (Wilkinson \& Pickett 2009). Consecuentemente es de esperar actitudes de autoritarismo, inseguridad y carencia de empatía, asociadas a la presencia de mayor delictividad, en particular a un índice más elevado de homicidios. Como lo señalan Wilkinson \& Pickett (2013), la desigualdad social "daña la calidad de las relaciones sociales” (pg. 60).

La criminalidad en avance en la sociedad peruana no encuentra una política que la combata y las explicaciones que se proponen (como aquella de que la delincuencia es una consecuencia del mayor desarrollo económico) generan indignación cuando no burla entre la opinión pública.

Por todo esto, cada día se escuchan en el Perú reclamos de una intervención más decidida del estado en el combate de la delincuencia y en la represión de la violencia que va asociada a los conflictos sociales. Se reclamó en algún momento, por ejemplo, la imposición del estado de emergencia, la supresión de beneficios penitenciarios, el restablecimiento de la pena de muerte y la aplicación de penas más severas, todo esto acompañado de críticas muy severas a la ineficiencia del estado, en particular del poder judicial, y a la corrupción de la policía.

Se trata por lo general de medidas represivas acompañadas de la demanda de la construcción de más cárceles para una población penal que no disminuye sino que, por el contrario, aumenta de año en año.

En todo esto se nota, sin embargo, la ausencia de una reflexión sostenida acerca de las causas de la delincuencia y, mucho más aún, de políticas de largo plazo que, unidas a acciones de efectividad inmediata, contribuyan a disminuir la delincuencia de modo permanente (Obando Morales-Bermúdez \& Ruíz Chipa 2007).

En este contexto se escuchan pocas voces que reclamen los derechos humanos. Algunas ONGs que los promueven son sometidas a duras criticas o se considera su acción como una intromisión en la soberanía nacional, cuando no como una defensa de los supuestos derechos de los terroristas.

Los resultados obtenidos permiten confirmar que los derechos humanos en el Perú, como dijimos, al comenzar la presente comunicación, tienen mala prensa, y los resultados obtenidos no parecen indicar que en un futuro cercano se puedan producir cambios en las actitudes de las personas sobre el particular. 


\section{DIRECCIONES}

1 Universidad Ricardo Palma - Facultad de Psicología. Email: rld310850@yahoo.com. $\mathrm{mx}$

2 Universidad Ricardo Palma - Facultad de Psicologia. Email: ej_gamarra_a@hotmail. com

\section{REFERENCIAS}

APOYO. Opinión y Mercado (2007). Opinión data. Resumen de encuestas a la opinión pública. Año 7, nro. 84 (22 de enero del 2007) .

BAIK, E. (2012). Gender, religión and national origin: Latinos`` attitude toward capital punishment. Journal of Social Science, 8 (1), 79-84.

BOLÍVAR, L. \& Cubas, R. (2009). Desarrollo del movimiento de derechos humanos en Venezuela durante los últimos 50 años. Caracas: Universidad Católica Andrés Bello (Centro de Derechos Humanos).

BRAVC, E.; Nicoletti, I.; Philipp, S. \& Starl, K. (2011). [Menschen]. Rechte. Bildung. Eine qualitative Evaluation von Menschenrechtsbildung in allgemeinbildenden höheren Schulen. Graz: Europäisches Trainings- und Forschungszentrum für Menschenrechte und Demokratie.

BROOKS, C. \& Manza, J. (2013). Whose rights? Counterterrorism and the dark side of American public opinion. New York: Russell Sage Foundation.

BURT, J.-M. (2006). "Quien habla es terrorista". The political use of fear in Fujimori" s Peru. Latin American Research Review, 41 (3), 32-62.

BURT, J.-M. (2010). Political violence and the authoritarian state in Peru: silencing civil societies. New York: Palgrave Macmillan.

CESARINI, P. \& Hertel, Sh. (2005). Interdisciplinary approaches to human rights scholarship in Latin America. Journal of Latin American Studies, 37, 793809.

Comisión de la Verdad y Reconciliación (2003). Informe Final. Lima.

DÍAZ-VEIZADES, J.: WIDAMAN, K. F.; LITTLE, T. D. \& GIBBS, K. W. (1995). The measurement and structure of human rights attitudes. Journal of Social Psychology, 135 (3), 313-328.

FLORES GALINDO, A. (1999). La tradición autoritaria. Violencia y democracia en el Perú. Lima: Aprodeh - Sur. 
HOWE, R. B. \& Covell, K. (2005). Empowering children: Children's rights education as a pathway to citizenship. Toronto: University of Toronto Press.

HUMPHREY, M. (2012). Citizen insecurity in Latin American cities: the intersection of spatiality and identity in the politics of protection. Critica Contemporánea: Revista de Teoría Política, nro. 2, noviembre, 1-18

IMSBUCH, P.; Misse, M.; Carrión, F. (2011). Violence research in Latin America and the Caribbean: a literature review. International Journal of Conflict and Violence, 5.1., 88-154.

INTERNATIONAL COUNCIL ON HUMAN RIGHTS POLICY (2003). Delincuencia, orden público y derechos humanos. Ginebra.

ISHAY, M. R. (2004). The history of human rights. From ancient times to the globalization era. Berkeley - Los Angeles: University of California Press.

ITURRALDE, M. (2010). Democracies without citizenship: crime and punishment in Latin America. New Criminal Law Review, 13 (2), 309-332.

KINDERMAN, P. (2007) Human rights and applied psychology. Journal of Community \& Applied Social Psychology, 17, 218-228.

LAY SUN, h. (2013). "El informe de la CVR en sí no va a reconciliar". El Comercio, edición del 25 de agosto, pg. A2 [entrevista].

LEÓN, R. (2010). Insultos y ofensas. Una reflexión acerca de las críticas a la persona de Mario Vargas Llosa. En: Rodríguez Rea, M. A., ed., Mario Vargas Llosa y la crítica peruana, Lima, Universidad Ricardo Palma, 543-576.

LERNER FEBRES, S. (2013). "La verdad de alguna manera se muestra y coloca las cosas en su lugar". El Comercio, edición del 28 de agosto; pg. A12 [entrevista].

LIPSET, S. M. (1959). Some social requisites of democracy: economic development and political legitimacy. American Political Science Review, 53 (1), 69-105.

LIPSET, S. M. (1960). Political man. New York: Doubleday.

LOVATÓN PALACIOS, D. \& Arias Aróstegui, E. (2010). Informe Anual 2010 sobre seguridad ciudadana. El legado del gobierno de Alan García: un país más inseguro. Lima: Instituto de Defensa Legal.

MAJLUF, A. (1989). Prácticas de crianza de madres de estratos socioeconómicos medio y bajo de Lima. Revista de Psicología, 7 (2), 151-161.

MAJLUF, A. \& Ojeda, G. (2006). Relación entre tradicionalismo y prácticas de crianza. Revista Psicológica Herediana, 1 (1), 28-38

MANRIQUE, N. (1996). Racismo y violencia política en el Perú. Pretextos, 8, 89-105.

MELÉNDEZ, C. (2013). Una verdad esquiva. Correo Semanal, edición del 29 de agosto, nro. 123, 24-27.

MESSER, E. (2002). Anthropology and human rights in Latin America. En: Heath, D., ed., Contemporary cultures and societies of Latin America, Prospect Heights, Ill., Wabeland Press, 18-30. 
MINISTERIO DE JUSTICIA Y DERECHOS HUMANOS (Consejo Nacional de Politica Criminal - Dirección General de Politica Criminal y Penitenciaria) (2013). La delincuencia en el Perú: propuesta de intervención articulada. Lima: Documento de Trabajo nro 01.

MIRELLA, F. (2011). El costo económico de la delincuencia organizada en el Perú: estudio sobre los costos directos que el crimen ocasiona al estado y las familias. Lima: Oficina de las Naciones Unidas contra la Droga y el Delito.

MOGHADDAM, F. M. \& Vuksanovic, V. (1990). Attitudes and behavior toward human rights across different contexts: the role of right-wing and religiosity. International Journal of Psychology, 25, 455-474.

NETTELBLADT, P.; Uddenberg, N; \& Englesson, I. (1981). Sex-role patterns, paternal rearing attitudes and child development in different social classes. Acta Psychiatrica Scandinavica, 64 (1), 12-24.

NOTICIA (2010). Alan García: "Hay ciertos crímenes que deben ser pagados con la vida". El Comercio, 17 de agosto.

NOTICIA (2012). Escritor Iván Thays critica a la comida peruana en España. El Comercio, 2 de febrero.

NOTICIA (2013). Aprobación del presidente [Humala] cae cinco puntos. El Comercio, ed. del 19 de mayo, pg. a4.

OBANDO MORALES-BERMÚDEZ, N. \& Ruíz Chipa, C. (2007). Determinantes socioeconómicos de la delincuencia: una primera aproximación al problema a nivel provincial. Informe final. Lima: CIES Consorcio de Investigación Económica y Social - CEDEP.

O`NEIL, K. M.; PATRY, M. C. \& PENROD, S. V. (2004). Exploring the effects of attitudes toward the death penalty on capital sentencing verdict. Psychology, Public Policy, and Law, 10 (4), 443-470.

OZBUDUN, E. (2005). The role of the middle class in the emergence and consolidation of a democratic civil society. Ankara Law Review, 2 (2), 95-107.

PANEZ, R. (1989). Bajo el sol de la infancia. Creencias y tradiciones en la crianza limeña. Lima: Concytec.

PARAMIO, L. (2012). Clases medias, política y democracia. Pensamiento Iberoamericano, 2da. época, 10, 275-293.

PEASE GARCÍA, H. \& Romero Sommer, G. (2013). La política en el Perú del siglo XX. Lima: Pontificia Universidad Católica del Perú.

PIRTTILÄ-BACKMAN, A-M.; Kassea, R. \& SAkki, I. (2009). Human and peoples' rights: social representations among Cameroonian students. International Journal of Psychology, 44, 459-467.

PORTOCARRERO, G. (1990). Violencia estructural en el Perú: sociología. Lima: Asociación Peruana de Estudios e Investigación para la Paz [con E. Acha como asistente de investigación].

PORTOCARRERO, G. (1993). Racismo y mestizaje. Lima: Sur. 
RAMÍREZ, F. O.; SUAREZ, D.; \& MEYER, J. W. (2006). The worldwide rise of human rights education: 1950-2005. En: Benavot, A. \& Braslavsky, C., eds., The changing contexts of primary and secondary education: comparative studies of the school curriculum (pp. 36-52). Hong Kong: CERC.

REYNA, C. \& TOCHE, E. (1999). La inseguridad en el Perú. Santiago, Chile: Naciones Unidas. Comisión Económica para América Latina y el Caribe.

RICO, J. M. \& CHINCHILLA, L. (2006). Las reforrnas policiales en América Latina: situación, problemas y perspectivas. Lima: Instituto de Defensa Legal.

SANTIVÁÑEZ VIVANCO, M. (2013). La iglesia y la Comisión de la Verdad. Correo, edición del 29 de agosto; pg. 4).

SANTILlANA, M. A. (2013). La verdad esquiva: Informe CVR. Correo, edición del 30 de agosto; pg. 9.

SARAT, A. \& Vidmar, N. (1976). Public opinion, the death penalty, and the Eight Amendment: testing the Marshall hyphotesis. Wisconsin Law Review, 1, $171,197$.

SHIRAEV, E. \& Sobel, R. (2006). People and their opinions. Thinking critically about public opinion. New York: Pearson Longman.

SUAREZ, D.; \& Ramírez, F. O. (2007). Human rights and citiuzenship: the emergence of human rights education. En: Torres, C., ed., Critique and utopia: new developments in the sociology of education (pp. 43-64). Lanham, MD: Rowman \& Littlefield.

TIANO, S. (1986). Authoritarianism and political culture in Argentina and Chile in the middle 1960s. Latin American Research Review, 21 (1), 73-98.

TORNEY-PURTA, J.; WILKENFELD, B.; BARBER, C. (2008). How adolescents in 27 countries understand, support, and practice human rights. Journal of Social Issues, 64, 857-880.

TORRES ARANCIVIA, E. (2003). Buscando un rey. El autoritarismo en la historia del Perú. Siglos XVI-XXI. Lima: Pontificia Universidad Católica del Perú.

VIII Encuesta Nacional sobre percepción de la corrupción en el Perú 2013. Lima.

WALKER, Ch. (2009). Diálogos con el Perú. Ensayos de historia. Lima: Fondo Editorial del Pedagógico San Marcos.

WILKINSON, R. \& Pickett, K. (2009). The spirit level: why more equal societies almost always do better. Londres: Allen Lane.

WILKINSON, R. \& Pickett, K. (2013). Sociedades disfuncionales: ¿por qué tiene importancia la desigualdad? En: El mundo de la clase media. Vanguardia Dossier, nro. 47, abril/junio, 59-63.

WRIGHT, Th. C. (2007). State terrorism in Latin America. Chile, Argentina, and international human rights. Lanham, Maryland: Rowman \& Littlefield. 


\section{ENCUESTA}

A continuación encontrarás 27 afirmaciones sobre la realidad social del Perú. Léelas, e indícanos si estás de acuerdo con ellas, según las siguientes posibilidades de calificación

$\underline{T A}=$ Totalmente de acuerdo $\underline{M A}=$ Muy de acuerdo

$\underline{\mathrm{DA}}=$ De acuerdo $\underline{\mathrm{NS}}=$ No tengo una opinión $\underline{\mathrm{ED}}=$ En desacuerdo $\underline{\mathrm{MD}}=$ Muy en desacuerdo $\underline{\mathrm{TD}}=$ Totalmente en desacuerdo

No hay respuestas buenas ni malas. La encuesta es anónima. Gracias por tu colaboración

Edad: .......... Sexo: Hombre Mujer Distrito de residencia: ..

\section{Tú te consideras alguien de}

Clase social alta __ Clase social media__ Clase social baja

En materia de politica tú te consideras de

Izquierda ....// ....// ....// ....// ....// ....// ....// ....// .... Derecha

\begin{tabular}{|c|c|c|c|c|c|c|c|c|}
\hline $\mathrm{Nr}$. & Afirmación & TA & MA & DA & NS & ED & MD & TD \\
\hline 01. & $\begin{array}{l}\text { La mayoria de los/as peruanos/as no está } \\
\text { preparada para vivir en democracia }\end{array}$ & TA & MA & DA & NS & ED & MD & $\mathrm{TD}$ \\
\hline 02. & $\begin{array}{l}\text { Debe aplicarse la pena de muerte para } \\
\text { delitos graves como la violación de niños }\end{array}$ & TA & MA & DA & NS & ED & MD & TD \\
\hline 03. & $\begin{array}{l}\text { Se habla demasiado de los derechos } \\
\text { humanos en el Perú }\end{array}$ & TA & MA & DA & NS & ED & MD & TD \\
\hline 04 & $\begin{array}{l}\text { Los mejores gobiernos que ha tenido el } \\
\text { Perú han sido militares }\end{array}$ & TA & MA & DA & NS & ED & MD & TD \\
\hline 05. & $\begin{array}{l}\text { Los diarios en el Perú muchas veces abusan } \\
\text { de la libertad de prensa }\end{array}$ & TA & MA & DA & NS & ED & MD & TD \\
\hline 06. & $\begin{array}{l}\text { Ante la ineficacia de la policia, muchas } \\
\text { veces está justificado que las personas } \\
\text { tomen la justicia por sus propias manos } \\
\end{array}$ & TA & MA & DA & NS & ED & MD & TD \\
\hline 07. & $\begin{array}{l}\text { En el Perú se debe tener mano dura para } \\
\text { controlar el desorden social }\end{array}$ & TA & MA & DA & NS & ED & MD & TD \\
\hline 08. & $\begin{array}{l}\text { Muchos peruanos/as abusan de la } \\
\text { democracia }\end{array}$ & TA & MA & DA & NS & ED & MD & TD \\
\hline 09. & $\begin{array}{l}\text { La policia debe reprimir con dureza los } \\
\text { desórdenes }\end{array}$ & TA & MA & DA & NS & ED & MD & $\mathrm{TD}$ \\
\hline 10. & $\begin{array}{l}\text { E1 Parlamento es una institución poco útil } \\
\text { para el Perú }\end{array}$ & TA & MA & DA & NS & ED & MD & TD \\
\hline 11. & $\begin{array}{l}\text { El estado debe controlar los contenidos de } \\
\text { los periódicos, la radio y la televisión }\end{array}$ & TA & MA & DA & NS & ED & MD & TD \\
\hline 12. & $\begin{array}{l}\text { En su gran mayoria los peruanos/as solo } \\
\text { respetan gobiernos autoritarios }\end{array}$ & TA & MA & DA & NS & ED & MD & TD \\
\hline 13. & $\begin{array}{l}\text { Para el Perú es más importante el } \\
\text { crecimiento económico que los derechos } \\
\text { humanos }\end{array}$ & TA & MA & DA & NS & ED & MD & TD \\
\hline 14. & \begin{tabular}{|l|} 
La libertad de prensa debe ser total en el \\
Perú
\end{tabular} & TA & MA & DA & NS & ED & MD & TD \\
\hline 15. & $\begin{array}{l}\text { Se debe legalizar las uniones del mismo } \\
\text { sexo en el Perú }\end{array}$ & TA & MA & DA & NS & ED & MD & TD \\
\hline
\end{tabular}




\begin{tabular}{|l|l|l|l|l|l|l|l|l|}
\hline 16. & Se debe legalizar el aborto en el Perú & TA & MA & DA & NS & ED & MD & TD \\
\hline 17. & $\begin{array}{l}\text { Los derechos humanos no deben valer para } \\
\text { los violadores de niños }\end{array}$ & TA & MA & DA & NS & ED & MD & TD \\
\hline 18. & $\begin{array}{l}\text { Las condenas judiciales a los terroristas } \\
\text { peruanos han sido muy benévolas }\end{array}$ & TA & MA & DA & NS & ED & MD & TD \\
\hline 19. & $\begin{array}{l}\text { Algunos politicos tienen como un negocio } \\
\text { suyo la defensa de los derechos humanos } \\
\text { sin pensar en lo que le conviene al pais }\end{array}$ & TA & MA & DA & NS & ED & MD & TD \\
\hline 20. & $\begin{array}{l}\text { El castigo fisico de los hijos por parte de } \\
\text { sus padres es un derecho de éstos y el } \\
\text { estado no debe intervenir }\end{array}$ & TA & MA & DA & NS & ED & MD & TD \\
\hline 21. & $\begin{array}{l}\text { A veces es necesario el castigo fisico en la } \\
\text { escuela }\end{array}$ & TA & MA & DA & NS & ED & MD & TD \\
\hline 22. & $\begin{array}{l}\text { El orden es lo más importante en una } \\
\text { sociedad }\end{array}$ & TA & MA & DA & NS & ED & MD & TD \\
\hline 23. & La autoridad siempre debe ser respetada & TA & MA & DA & NS & ED & MD & TD \\
\hline 24. & $\begin{array}{l}\text { Las organizaciones que promueven los } \\
\text { derechos humanos en el Perú (ONGs) } \\
\text { muchas veces defienden solo a terroristas y } \\
\text { no a sus victimas }\end{array}$ & TA & MA & DA & NS & ED & MD & TD \\
\hline 25. & Debe haber pena de muerte en el Perú & TA & MA & DA & NS & ED & MD & TD \\
\hline 26. & Los jueces acá son muy “blandos" & TA & MA & DA & NS & ED & MD & TD \\
\hline 27. & $\begin{array}{l}\text { Los delincuentes tienen demasiados } \\
\text { derechos en el Perú (por ejemplo, } \\
\text { reducción de pena) }\end{array}$ & TA & MA & DA & NS & ED & MD & TD \\
\hline
\end{tabular}

\section{Calificación de los items}

1 a 13: $T D=1$ TA= 7

14 a 16: $T A=1$ TD $=7$

17 a 27: $T D=1$ TA=7 